\title{
Combined Use of a High-Density Multi-electrode Mapping Catheter and a Magnetically Navigated Ablation Catheter for Atrial Fibrillation Procedures: A Feasibility Study
}

\author{
BURKHARD HÜGL, MD ${ }^{1}$, BJOERN BUCHTER, MD ${ }^{1}$, ZDRAVENA DONEVA, MD ${ }^{1}$, ARNE GEISSLER, MD ${ }^{1}$, \\ LEILANI SELL, MD ${ }^{1}$, TAYLOR TSO, BS ${ }^{2}$, STEFAN MIRAZCHIYSKI, BS ${ }^{2}$ and QUN SHA, MD ${ }^{2}$ \\ ${ }^{1}$ Marienhaus Klinikum Neuwied, Kardiologie/Rhythmologie, Neuwied, Germany \\ ${ }^{2}$ Stereotaxis Inc., St. Louis, MO
}

\begin{abstract}
PentaRay ${ }^{\circledR}$ catheters (Biosense Webster, Diamond Bar, CA) create high-density electroanatomical maps, and remote magnetic navigation (RMN) (Niobe Epoch ${ }^{\mathrm{TM}}$, Stereotaxis Inc., St. Louis, MO) enables intricate manipulation of magnetic ablation catheters. The purpose of this study was to assess the feasibility of PentaRay ${ }^{\circledR}$ used in combination with RMN ablation for pulmonary vein isolation (PVI). In 21 paroxysmal atrial fibrillation (AF) patients undergoing PVI, a PentaRay ${ }^{\circledR}$ catheter was used to map the left atrium in sinus rhythm at baseline and post PVI. The Navigant Ablation History module (Stereotaxis Inc., St. Louis, MO) was used to help create connected ablation lines, pacing from the ablation catheter (ThermoCool RMT, Biosense Webster, Diamond Bar, CA) was performed to examine the presence of exit block, and voltage maps from the PentaRay ${ }^{\circledR}$ were used to demarcate low-voltage areas where bipolar voltages were within $0.25-0.5$ $m V$. This information was assessed to identify potential PVI gaps, and additional ablation points were delivered as necessary. Complete PVI was achieved in 21 out of 21 patients (100\%). The average initial mapping time was $12.4 \pm 2.7$ min spanning $1,726 \pm 476$ points. Similarly, the average remap time was $14 \pm 4.3$ min spanning $1,928 \pm 842$ points. There were no procedural complications associated with this study. This study demonstrates the feasibility of this clinical workflow using both a high-density multi-electrode mapping catheter and an RMN ablation catheter for PVI. The combination might be a useful strategic choice for treatment of AF.
\end{abstract}

KEYWORDS. ablation, atrial fibrillation, high-density mapping, remote magnetic navigation.
ISSN 2156-3977 (print) ISSN 2156-3993 (online)

(C) 2016 Innovations in Cardiac Rhythm Management

\footnotetext{
Stefan Mirazchiyski has a financial relationship with one or more companies that produce or market products or services relevant to the topic of this manuscript: Stereotaxis Inc., Account Manager Clinical Adoption. Qun Sha and Taylor Tso have financial relationships with one or more companies that produce or market products or services relevant to the topic of this manuscript: Stereotaxis Inc., Employee. All other authors report no conflicts of interest for the published content.

Manuscript received May 16, 2016, Final version accepted June 20, 2016.

Address correspondence to: Burkhard Hügl, Marienhaus Klinikum, St. Elisabeth Neuwied, Department Cardiology, Friedrich-Ebert-Str. 59, 56564, Neuwied, Germany.

E-mail: burkhard.huegl@marienhaus.de
}

\section{Introduction}

Pulmonary vein isolation (PVI), using manual or magnetic navigation-guided radiofrequency (RF) ablation, is the common approach to treat patients with symptomatic paroxysmal and persistent atrial fibrillation (AF). AF recurrence is a frequent outcome, however, and patients often undergo repeat ablations after conventional PVI procedures. Currently, PVI is frequently performed with a circular mapping catheter (CMC). Although a CMC can quickly acquire anatomical and electrogram data around the pulmonary veins (PVs), its electrode configuration is not conducive to mapping elsewhere in the atria. 
Atrial low-voltage areas (LVAs) have been defined elsewhere $^{1-4}$ where electrogram amplitudes are $\leq 0.5 \mathrm{mV}$, and their presence has been shown to correlate with impaired outcomes after PVI. Since LVAs are associated with atrial scar and/or structural defects, substratebased modification via targeting LVAs in PV and non-PV areas of the left atrium may be an important therapeutic strategy to maximize long-term AF freedom. ${ }^{1,2,5}$

The PentaRay ${ }^{\circledR}$ (Biosense Webster, Baldwin Park, CA) is a high-density multipolar electrode mapping catheter capable of simultaneously acquiring multiple voltage data points. The main advantage of this novel mapping catheter is the atraumatic splaying of all five splines against the endocardial surface, providing a high-density map from its $201-\mathrm{mm}$ electrodes covering a surface of more than $7 \mathrm{~cm}^{2}$. Compared with point-by-point voltage maps, PentaRay ${ }^{\circledR}$ maps have been shown to have significant advantages such as less mapping time and greater spatiotemporal resolution. ${ }^{4}$

Remote magnetic navigation (RMN)-guided RF ablation using the Niobe ES system (Stereotaxis Inc., St. Louis, $\mathrm{MO}$ ) allows the physician to precisely position the ablation catheter in a specific endocardial location remotely from a control room. Numerous clinical advantages are associated with the RMN system, ${ }^{6}$ which has an excellent safety profile because of its flexible magnetic catheter navigated by virtue of magnetic pulling forces rather than the pushing contact forces associated with manual catheters. The RMN modality also provides stable, constant catheter contact during ablation, which may allow for more efficacious therapy delivery. ${ }^{7,8}$

The purpose of this study was therefore to investigate the feasibility of using an atraumatic PentaRay ${ }^{\circledR}$ catheter for high-density mapping in conjunction with an atraumatic magnetic catheter for PVI in AF patients. This is the first study of PentaRay ${ }^{\circledR}$ mapping catheters used in conjunction with RMN technology.

\section{Methods}

\section{Study population}

Consecutive patients undergoing first-time PVI for paroxysmal AF between March 2014 and February 2015 were included in this study. Patients with previous catheter ablation or cardiac surgery were excluded. Eligible subjects were $\leq 80$ years old and presented with paroxysmal AF with no valvular disease. The ethics committee of Marienhaus Klinikum St. Elisabeth, Neuwied, Germany, approved the study protocol, and subjects provided written informed consent before the ablation procedure. Patient characteristics including age, comorbidities, and gender were recorded upon successful study enrollment.

\section{General procedure set-up}

Patients were studied under deep sedation. After transseptal puncture and insertion of an SL1 sheath
(St. Jude Medical, Memphis, TN), a PentaRay ${ }^{\circledR}$ catheter and a 4-mm-tip irrigated ablation catheter (ThermoCool RMT, Biosense Webster, Diamond Bar, CA) were placed into the left atrium. After establishing left atrial access, intravenous heparin was administered with a target activated clotting time of $300 \mathrm{~s}$.

\section{High-density electroanatomical mapping}

A detailed bipolar left atrium (LA) voltage map was acquired during sinus rhythm using a PentaRay ${ }^{\circledR}$ catheter with a CARTO 3 mapping system (Biosense Webster, Diamond Bar, CA). The PentaRay ${ }^{\circledR}$ multielectrode mapping catheters for this study had 1-mm electrodes with interelectrode spacings of $4-4-4 \mathrm{~mm}$. Bipolar voltages were recorded between the first and second electrode pairs and the third and fourth pairs on each spline. All bipolar electrograms were filtered between 30 and $500 \mathrm{~Hz}$. LVAs were identified as regions where voltages were within $0.25-0.5 \mathrm{mV}$. All maps were also checked within the range of $0.05-0.50 \mathrm{~V}$.

\section{Magnetic navigation-guided catheter ablation and electroanatomical remapping}

Ablation was performed with the ablation catheter controlled via RMN. The standard ablation settings consisted of an upper temperature limit of $43^{\circ} \mathrm{C}$, an $\mathrm{RF}$ power setting of $35 \mathrm{~W}$, a flow rate of $30 \mathrm{ml} / \mathrm{s}$, and an upper duration limit of $30 \mathrm{~s}$ per ablation point. The ablation strategy was circumferential PVI with bidirectional conduction block as an endpoint as determined by ThermoCool RMT ${ }^{\circledR}$ (Biosense Webster, Baldwin Park, CA) ablation catheter interrogation. The Navigant Ablation History module (Stereotaxis Inc., St. Louis, MO) was used to help create connected ablation lines. LVAs identified in the PV regions were also targeted for ablation. Following circumferential PVI and substrate modification, another detailed bipolar voltage map was created during sinus rhythm using the PentaRay ${ }^{\circledR}$ to assess for potential gaps. Any potential reconnection sites identified during this map were targeted for additional ablation.

\section{Statistical analysis}

Continuous measurements are presented as mean \pm standard deviation. Categorical measurements are presented as number positive for the condition and percentage of the total.

\section{Results}

\section{Patient characteristics}

A total of 21 patients with paroxysmal AF were included in the study. Patient characteristics are shown in Table 1. Minimal areas of fibrosis were detected in four patients via electroanatomical mapping. The ejection fractions (EFs) in all patients were above 55\% except for one patient whose EF was $40 \%$ (Table 1). 
Table 1: Patient characteristics

\begin{tabular}{lc}
\hline & All patients $(\mathbf{N}=\mathbf{2 1})$ \\
\hline Male, $\mathrm{n}(\%)$ & $15(71)$ \\
Age, years & $59 \pm 12$ \\
Height, cm & $175 \pm 10$ \\
Weight, kg & $88 \pm 15$ \\
Cardiomyopathy, $\mathrm{n}(\%)$ & $5(24)$ \\
Hypertension, $\mathrm{n}(\%)$ & $16(76)$ \\
Renal failure, $\mathrm{n}(\%)$ & $4(19)$ \\
Fibrosis, $\mathrm{n}(\%)$ & $4(19)$ \\
Left atrial diameter, mm & $3.9 \pm 0.6$ \\
Left ventricular ejection fraction, \% & $58 \pm 5$ \\
\hline
\end{tabular}

All values are rounded to nearest whole number except the left atrial diameter, which is rounded to the nearest tenth.

\section{Procedural outcomes}

Complete PVI was achieved in all 21 patients. Procedural parameters are listed in Table 2 . The ablation procedure lasted between $92.8 \mathrm{~min}$ and $163.2 \mathrm{~min}$, spanning between 91 and 150 burns across all patients. There were no clinical differences shown between voltage maps with $0.05 \mathrm{~V}$ or $0.25 \mathrm{~V}$ as the lower cutoff for defining LVAs. An example of ablation history is shown in Figure 1, and
Table 2: Procedural parameters

\begin{tabular}{lc}
\hline & All patients $(\mathbf{N}=\mathbf{2 1})$ \\
\hline Initial map duration, min & $12.4 \pm 2.7$ \\
Initial map points, $\mathrm{n}$ & $1726.2 \pm 475.6$ \\
Initial map fluoroscopy time, $\min$ & $5.1 \pm 1.7$ \\
Initial map fluoroscopy dose, cGy $\mathrm{cm}^{2}$ & $732.4 \pm 474.6$ \\
Ablation duration, min & $131.0 \pm 17.1$ \\
Ablation points, $\mathrm{n}$ & $125.4 \pm 16.0$ \\
Remap duration, min & $14 \pm 4.3$ \\
Remap points, $\mathrm{n}$ & $1927.9 \pm 841.9$ \\
Remap fluoroscopy time, $\min$ & $4.8 \pm 1.7$ \\
Remap fluoroscopy dose, $\mathrm{cGy} \cdot \mathrm{cm}^{2}$ & $774 \pm 48$ \\
\hline
\end{tabular}

All values are rounded to the nearest tenth.

electroanatomical mapping/remapping results are shown for the same patient in Figure 2. No patients required additional ablation as a result of identified gaps in the remap (Table 2 ).

\section{Complications}

There were no procedural complications associated with the 21 patients in this study.

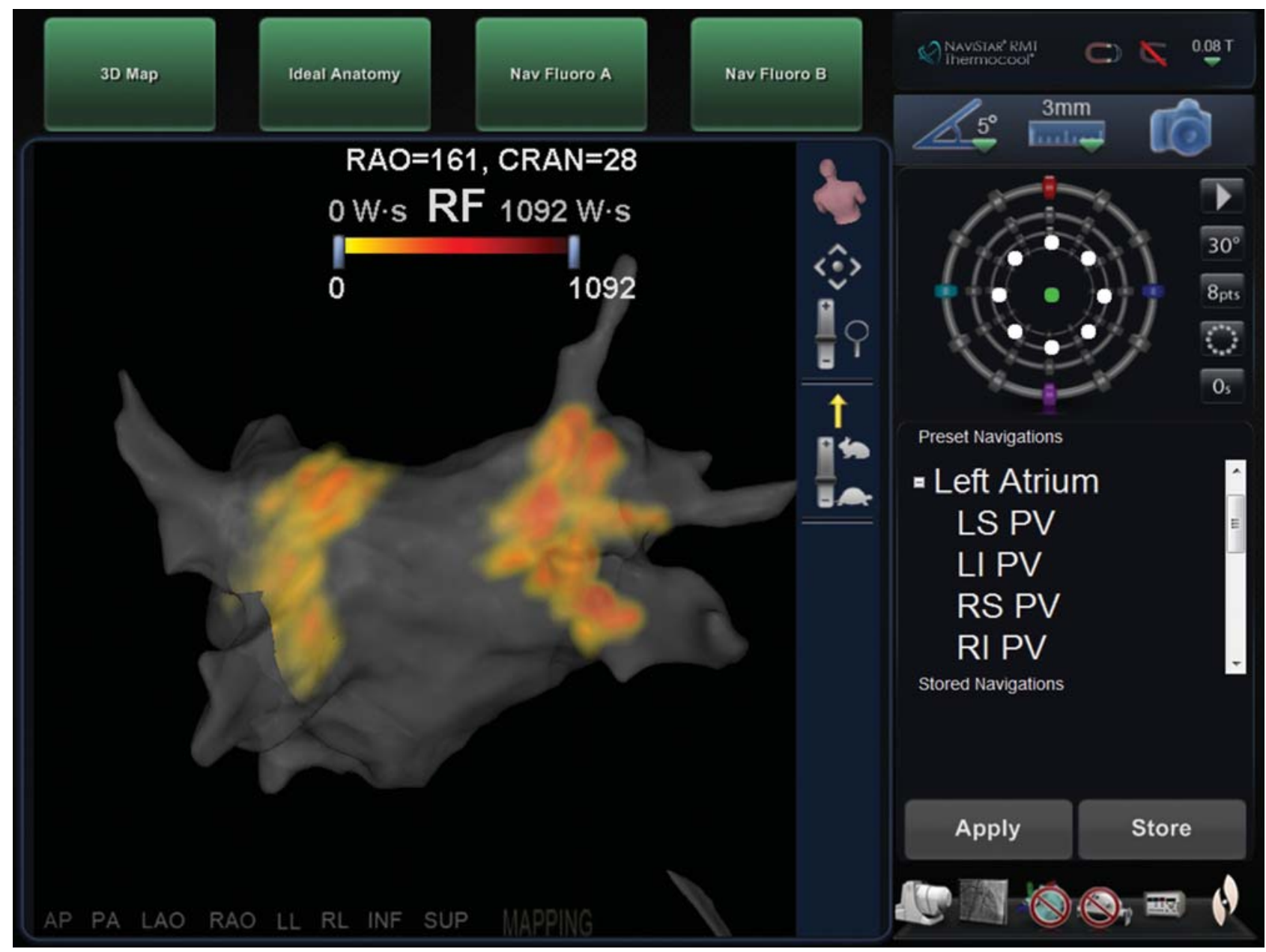

Figure 1: Navigant ablation history displays total W.s applied during the procedure. It was used to assess ablation lines for potential gaps prior to electroanatomical remapping. 


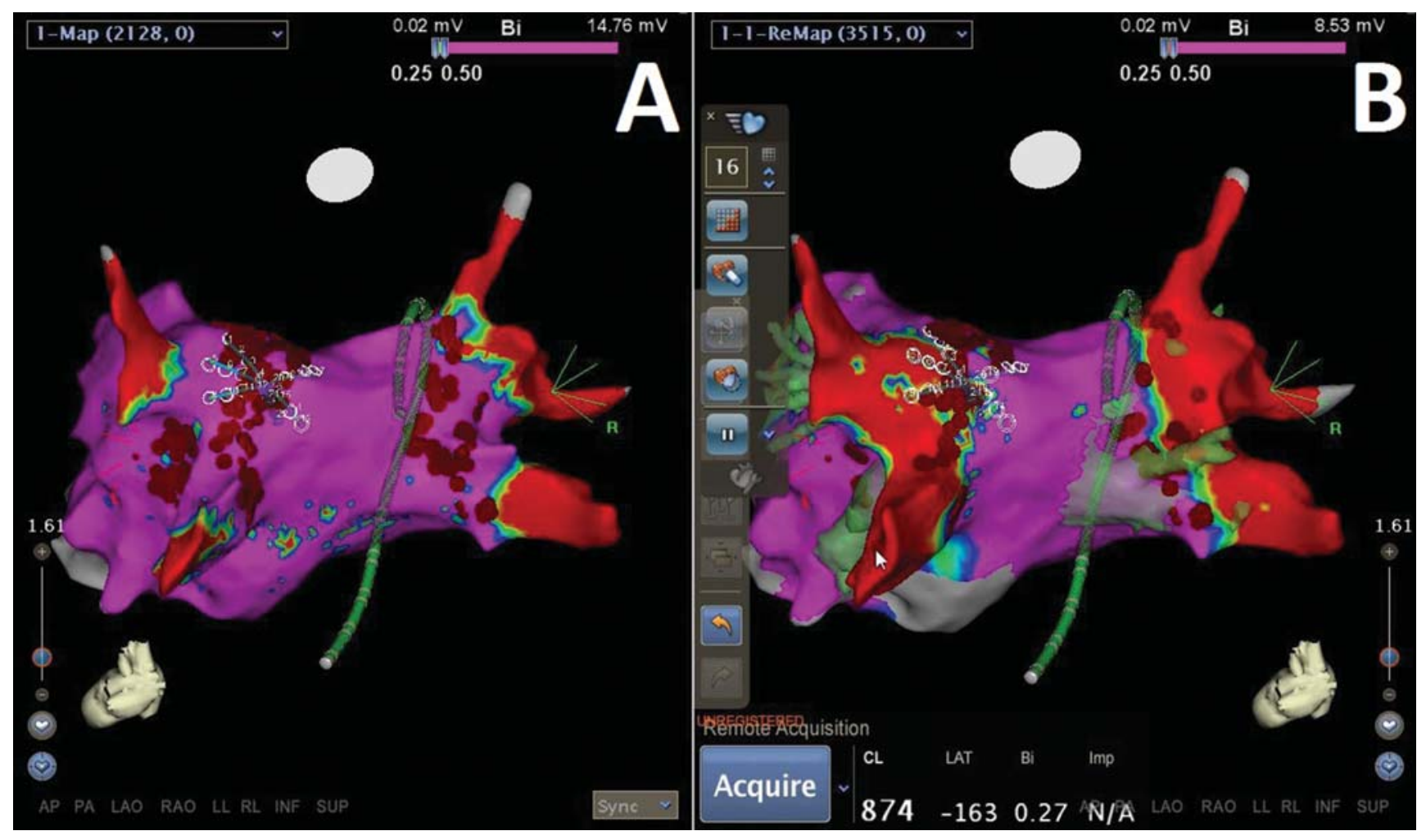

Figure 2: Bipolar voltage distribution in a patient's left atrium before (a) and after (b) pulmonary vein isolation. The voltage maps were created in sinus rhythm using a range of $0.25-0.50 \mathrm{mV}$ and are displayed in the posterior projection.

\section{Discussion}

This study demonstrates the feasibility of using a highdensity PentaRay ${ }^{\circledR}$ mapping catheter to delineate LVAs in patients undergoing remote magnetically guided RF ablation therapy for paroxysmal AF. The acute procedural endpoints were achieved in all subjects without complication, and the PentaRay ${ }^{\circledR}$ remap confirmed electrical isolation without identifying any gaps across all patients. These efficacious ablation lines may have been in part due to the ablation history allowing physicians to visualize the general shape of total RF applications. The ablation procedure lasted longer than $2 \mathrm{~h}$ on average. This relatively long duration is likely attributable to the substrate modification performed in addition to PVI. No gaps were identified in the PentaRay ${ }^{\circledR}$ remap, possibly because of this extensive ablation approach.

Currently, AF ablation procedures are often performed with a CMC, which is positioned within the PVs to assess for entrance and exit block. A CMC can acquire anatomical and electrogram data quickly around a PV, although some PVs are too small to enter. CMCs have also been used for high-density mapping in the LA, in particular using novel algorithms such as automatic point acquisition and impedance-based proximity sensing. The PentaRay ${ }^{\circledR}$ catheter facilitated an accurate and fast construction of the left atrial map. The initial map guided us to the pulmonary vein and quickly identified it as the source of the arrhythmia. The PentaRay ${ }^{\circledR}$ allowed us to take more than 1,700 points in less than
$13 \mathrm{~min}$ and formulate a single map when other technologies would have taken longer to achieve the same result. We used PentaRay ${ }^{\circledR}$ catheters with 4-4-4 mm electrode configurations allowing for an evenly distributed spatial resolution along each spline at the expense of far-field interference. Other PentaRay ${ }^{\circledR}$ catheters using 2-6-2 mm electrode configurations would be expected to provide more detailed local electrogram (EGM) information by increasing the distance between electrode pairs. Future studies should investigate which electrode configuration is most useful.

LVAs in the left atrium have recently been considered potential ablation targets. They are associated with endocardial fibrosis or scarring and have been shown to result in impaired outcomes after PVI. Rolf et $\mathrm{al}^{3}$ reported that LVAs outside the encircled PVs appeared in $10 \%$ of paroxysmal AF (PAF) patients and $35 \%$ of persistent atrial fibrillation (persAF) patients out of 178 total AF patients, preferentially located in the septal, anterior, and posterior left atrium. Furthermore, patients with LVAs who underwent strategic substrate modification targeting the LVAs had a better arrhythmia-free survival rate after 1 year. ${ }^{3}$ Electroanatomical mapping using PentaRay ${ }^{\circledR}$ is a viable modality to facilitate this substrate modification.

In a recent single-center evaluation of 233 patients with symptomatic paroxysmal AF, Bastian et al. ${ }^{9}$ demonstrated that RMN-guided PVI procedures are associated with reduced radiation exposure $\left(2.5 \pm 3.4 \mathrm{~Gy} \cdot \mathrm{cm}^{2}\right)$ and slightly longer procedure durations (300 $\pm 90 \mathrm{~min})$. 
Since this was a feasibility study and all operators experienced a learning curve with the novel workflow, fluoroscopy exposure and total procedural time tended to be higher. However, it is reasonable to expect that these parameters will be optimized alongside a streamlined workflow and a practiced physician.

Acute procedural success of RF ablations and durable lesion formation often depend on effective and safe mapping and ablation combination strategies. Both mapping and ablation workflows are crucial for procedural success. To our best knowledge, the study is the first clinical report based on the combined use of these novel technologies-mapping using PentaRay ${ }^{\circledR}$ and ablation using magnetic navigation. Magnetic navigation in conjunction with high-density mapping is a versatile approach facilitating RF ablation to treat AF. Future studies should explore any clinical benefit this combination approach might offer toward PVI and non-PV ablation procedures.

\section{Limitations}

This study was a non-randomized observational study that included a relatively small cohort of patients. A CMC was not used to verify PVI, and only acute procedural endpoints were analyzed. Future studies should assess the long-term clinical benefits of the combined approach described herein.

\section{Conclusion}

Our study demonstrates that it is effective and safe to use a PentaRay ${ }^{\circledR}$ mapping catheter to delineate LVAs in patients undergoing magnetically guided RF ablation therapy for paroxysmal AF. Although the report was a feasibility study in nature, the effective clinical workflow in AF ablations using both a high-density mapping catheter and an RMN ablation catheter could be an effective strategy to enhance ablation outcomes by targeting LVAs.
Acknowledgements. Qun Sha, MD is at present affiliated with Biosense Webster, Diamond Bar, CA.

\section{References}

1. Verma A, Wazni OM, Marrouche NF, et al. Pre-existent left atrial scarring in patients undergoing pulmonary vein antrum isolation: An independent predictor of procedural failure. J Am Coll Cardiol. 2005;45(2):285-292.

2. Rolf S, Hindricks G, Sommer P, et al. Electroanatomical mapping of atrial fibrillation: Review of the current techniques and advances. J Atr Fibrillation. 2014;7(4):58-68.

3. Rolf S, Kircher S, Arya A, et al. Tailored atrial substrate modification based on low-voltage areas in catheter ablation of atrial fibrillation. Circ Arrhythm Electrophysiol. 2014;7(5):825-833.

4. Anter E, Tschabrunn CM, Josephson ME. High-resolution mapping of scar-related atrial arrhythmias using smaller electrodes with closer interelectrode spacing. Circ Arrhythm Electrophysiol. 2015;8(3):537-545.

5. Kapa S, Desjardins B, Callans DJ, Marchlinski FE, Dixit S. Contact electroanatomic mapping derived voltage criteria for characterizing left atrial scar in patients undergoing ablation for atrial fibrillation. J Cardiovasc Electrophysiol. 2014;25(10):1044-1052.

6. Bradfield J, Tung R, Mandapati R, Boyle NG, Shivkumar K. Catheter ablation utilizing remote magnetic navigation: A review of applications and outcomes. Pacing Clin Electrophysiol. 2012;35(8):1021-1034.

7. Davis DR, Tang AS, Gollob MH, Lemery R, Green MS, Birnie DH. Remote magnetic navigation-assisted catheter ablation enhances catheter stability and ablation success with lower catheter temperatures. Pacing Clin Electrophysiol. 2008;31(7):893-898.

8. Thornton AS, De Castro CA, van Deel E, van Beusekom $\mathrm{HM}$, Jordaens L. An in vivo comparison of radiofrequency cardiac lesions formed by standard and magnetically steered $4 \mathrm{~mm}$ tip catheters. Neth Heart J. 2010;18(2):66-71.

9. Bastian D. Minimizing radiation exposure using remote magnetic catheter navigation for pulmonary vein isolation in patients with paroxysmal atrial fibrillation (Abstracts of the 11th Annual Congress of the European Cardiac Arrhythmia Society). J Interv Card Electrophysiol. 2015; 42(3):173-326. Abstract. 